\title{
The 4-parameter Compressible Packing Model (CPM) including a critical cavity size ratio
}

\author{
Gerard Roquier ${ }^{1, *}$ \\ ${ }^{1}$ Laboratoire Navier, 2 allée Kepler, Cité Descartes, 77420 Champs-sur-Marne (France). \\ gerard.roquier@laposte.net
}

\begin{abstract}
The 4-parameter Compressible Packing Model (CPM) has been developed to predict the packing density of mixtures constituted by bidisperse spherical particles. The four parameters are: the wall effect and the loosening effect coefficients, the compaction index and a critical cavity size ratio. The two geometrical interactions have been studied theoretically on the basis of a spherical cell centered on a secondary class bead. For the loosening effect, a critical cavity size ratio, below which a fine particle can be inserted into a small cavity created by touching coarser particles, is introduced. This is the only parameter which requires adaptation to extend the model to other types of particles. The 4-parameter CPM demonstrates its efficiency on frictionless glass beads (300 values), spherical particles numerically simulated (20 values), round natural particles (125 values) and crushed particles (335 values) with correlation coefficients equal to respectively $99.0 \%, 98.7 \%, 97.8 \%, 96.4 \%$ and mean deviations equal to respectively $0.007,0.006,0.007,0.010$.
\end{abstract}

\section{Introduction}

The packing density is often an optimized physical value for high performing and sustainable materials. Packing density models call upon to geometrical interaction coefficient functions of the size ratio: the wall effect $b$ and the loosening effect $a$ [1-7] to which can be added $[2-3,5]$ a wedging effect $c$. Because the packing density depends on the packing process efficiency and on the particle shape, two strategies can be adopted.

The first strategy $[2-3,5]$ consists in taking that into account in the mathematical expressions used for the interaction coefficients. It means that an adjustment by regression analysis is each time necessary to find the best functions of these parameters. Three sets have been proposed by the authors: the first one for the spherical particles, the second and the third ones for respectively the compacted and uncompacted angular particles. Recently, the interaction function for the wedging effect $c$ has been expressed in two separated parts [5].

The second strategy $[4,7]$ consists in taking into account of the packing process efficiency by a compaction index $K$ and of the particle shape and of the surface texture through a critical cavity size ratio $x_{0}$. Below this value, a small particle can be inserted into a small cavity created by touching coarser particles without disturbed their arrangement. Beyond this value, the local volume of the coarse class decreases: a loosening effect occurs. Thus, the 4-parameter Compressible Packing Model (CPM) [4, 7] has been developped by incorporating an original theory about the wall effect $b$ and the loosening effect $a$. The 4 parameters are: $a, b, K$ and $x_{0}$. Even if the expressions of $a$ and $b$ are more sophisticated, the adjustment of $K$ and $x_{0}$ has proven effective for spherical particles for which $x_{0}=0.2$, in harmony with the tetrahedral cavern theory $\left(x_{0} \approx 0.224\right)$. It remains to be able to adjust easily $x_{0}$ for other types of particles, especially angular ones.

\section{Wall effect, loosening effect, interference effect}

Fig. 1 represents the "void ratio" $\left(\mathrm{e}=1 / \phi^{*}-1\right)$ of a binary mixture $v s$ the "volume fraction of fine particles". In the "coarse dominant" field, the insertion mechanism is represented by AM and, in the "fine dominant" field, the substitution mechanism by FM. The wall effect is highlighted by FH which is located above FO. In the AK section, the loosening effect is only localized around a particle insufficiently fine to insert into a cavity created by some touching coarser particles. The more global interference effect is materialized by the AKJI section.

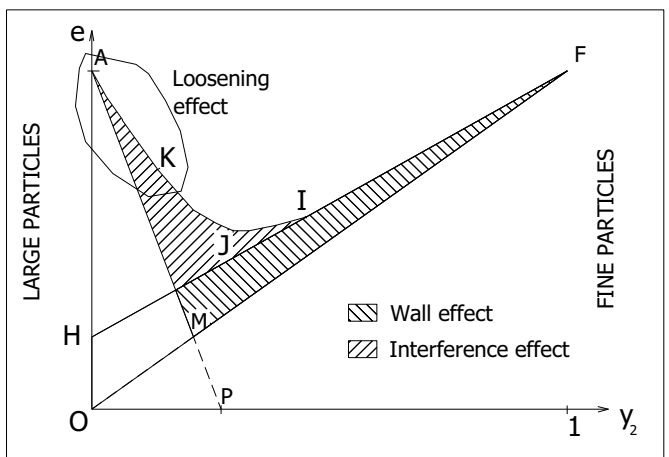

Fig. 1. Illustration of wall effect, loosening effect, interference effect. 


\section{The 4-parameter CPM}

The reference frame is constituted by the CPM [1]. The real packing density corresponds to that obtained for a randomly dense packing. It depends on the process used to fill and to compact the material inside the mould. If we consider a perfect placing process where each particle is placed one by one in its ideal location, the compaction index $K$ tends to infinity and the packing density reaches the virtual packing density. For a binary mixture "with geometrical interactions", the virtual packing density $\gamma$ obtained by the CPM can be written:

$$
\gamma=\operatorname{Min}\left(\gamma_{1}=\frac{\beta_{1}}{1-\left(1-\frac{\beta_{1}}{\beta_{2}} a_{12}\right) y_{2}} ; \gamma_{2}=\frac{\beta_{2}}{1-\left(1-\beta_{2}+b_{21} \beta_{2}\left(1-\frac{1}{\beta_{1}}\right)\right) y_{1}}\right) \text { (1) }
$$

where $\gamma_{1}$ and $\gamma_{2}$ are respectively the virtual packing densities of the binary mixture in the "coarse dominant" field and in the "fine dominant" field, $\beta_{1}$ and $\beta_{2}$ the virtual packing densities of the coarse size class 1 and of the fine size class 2, $y_{1}$ and $y_{2}$ their volume fractions by reference of the total solid volume, $a_{12}$ the loosening effect coefficient, $b_{21}$ the wall effect coefficient. When the size ratio $x=\mathrm{d}_{2} / \mathrm{d}_{1}$ (fine/coarse) is equal to 1 , a total interaction occurs:

$$
b_{21}(1)=1 \text { and } a_{12}(1)=1
$$

The real packing density $\phi^{*}$ is calculated by introducing the compaction index $K$. The expression (3) is an implicit equation of $\phi^{*}$, with one and only one positive root.

$$
K=\frac{\frac{y_{1}}{\beta_{1}}}{\frac{1}{\phi^{*}}-\frac{1}{\gamma_{1}}}+\frac{\frac{y_{2}}{\beta_{2}}}{\frac{1}{\phi^{*}}-\frac{1}{\gamma_{2}}}
$$

\subsection{Wall effect theory for spheres}

The wall effect is the further amount of voids in the packing of small particles against the wall of a coarser one. The disturbance is studied in a portion delimited by two concentric spheres. The first one has a diameter $d_{1}$. The second hypothetical one is chosen in such a way that the boundary condition $b_{2 l}(1)=1$ is respected. That's why we call this theory: ROAD (ROund ADjustable cell) theory.

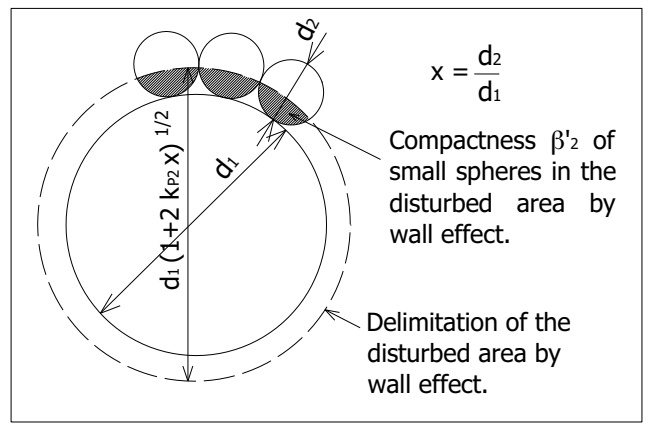

Fig. 2. Definition of the spherical reference cell for studying the wall effect.
The diameter of the sphere delimiting the disturbed volume by the wall effect around a coarse sphere is:

$$
d_{h y p}=d_{1} \sqrt{1+2 k_{P 2} x}
$$

Let $\alpha_{2}$ be the real packing density of the fine class 2 . We can deduce its virtual packing density $\beta_{2}$ by involving $K$ :

$$
\beta_{2}=\alpha_{2} \frac{(1+K)}{K}
$$

The number of fine spheres against a coarse one is calculated from the Spherical Square Model (SSM), not presented here, as part of the dense virtual packings:

$$
N_{12, S S M}^{\text {dense }}(x)=\frac{\pi(1+x)}{x \arcsin \left(\frac{x}{1+x}\right)}
$$

The packing density $\beta_{2}^{\prime}$ of the fine particles in the disturbed volume by the wall effect is then deduced:

$$
\begin{aligned}
& \beta_{2}^{\prime}(x)=\frac{\pi(1+x)}{4 x\left(\left(1+2 k_{P 2} x\right)^{\frac{3}{2}}-1\right) \arcsin \left(\frac{x}{1+x}\right)} \times \\
& \times\left(2\left(1+2 k_{P 2} x\right)^{\frac{3}{2}}-3\left(1+2 k_{P 2} x\right)\left(1+\frac{k_{P 2} x}{1+x}\right)+\left(1+\frac{k_{P 2} x}{1+x}\right)^{3}-x^{3}+\frac{3 k_{P 2} x^{3}}{1+x}+x^{3}\left(1-\frac{k_{P 2}}{1+x}\right)^{3}\right)
\end{aligned}
$$

In the case where $\beta_{1}=\beta_{2}$, the wall effect coefficient is:

$$
b_{21}(x)=\frac{\left(\beta_{2}-\beta_{2}^{\prime}(x)\right)}{\left(1-\beta_{2}\right)}\left[\left(1+2 k_{P 2} x\right)^{\frac{3}{2}}-1\right]
$$

It remains to be determined $k_{P 2}$ by respecting the boundary condition $b_{2 I}(1)=1$, leading to the numerical solution, of the following equation:

$$
\left(\beta_{2}-6\right)\left(1+2 k_{P 2}\right)^{\frac{3}{2}}+\frac{9}{2} k_{P 2}^{2}+18 k_{P 2}+5=0
$$

The equation to be solved being of the $3^{\text {rd }}$ degree, the value of $\mathrm{k}_{\mathrm{P} 2}$ which is coherent with those presented in the following table should be kept:

Table 1. Examples of values of $k_{P}$ as a function of the virtual packing density $\beta$.

\begin{tabular}{|l|c|c|c|c|c|}
\hline $\boldsymbol{\beta}$ & 0.65 & 0.68 & 0.70 & 0.72 & 0.734 \\
\hline $\boldsymbol{k}_{\boldsymbol{P}}$ & 0.4466 & 0.7543 & 0.9253 & 1.1476 & 1.4729 \\
\hline
\end{tabular}

An example of the wall effect coefficient $b_{21}$ as a function of the size ratio $x$ is given Fig. 3. The results from the ROAD theory have been achieved for a compaction index $K=9$ corresponding to a packing process "vibration + compression" (Table 2) and a real packing density of the fine class $2 \alpha_{2}=0.641$ corresponding to the Random Close Packing RCP state. 


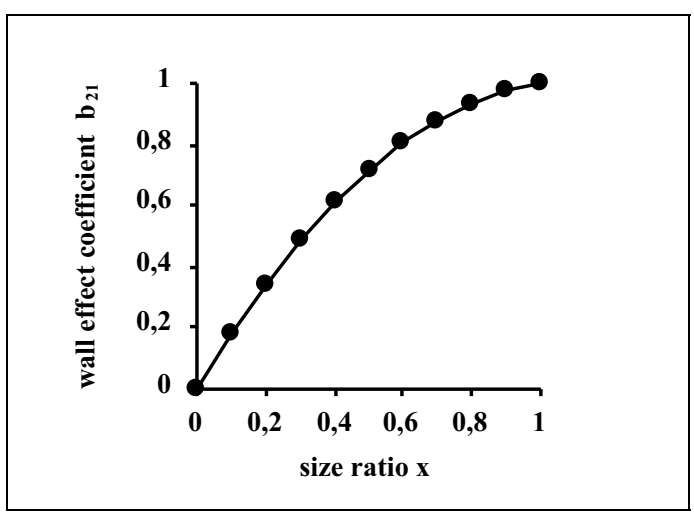

Fig. 3. ROAD theory: $\alpha_{2}=0.641-$ Vibration + compression.

\subsection{Loosening effect and interference effect theory for spheres}

The concept of critical cavity size ratio $x_{0}$ is now introduced: below this value, the intrusion of small quantities of finer spheres does not disturb the bed of coarser spheres; beyond this value, the fine spheres cannot be placed without disturbing this one. The concept of local isotropic expansion of the coarse particle skeleton around a small one is adopted when $x>x_{0}$. To be consistent with the wall effect theory, a spherical reference cell is used.

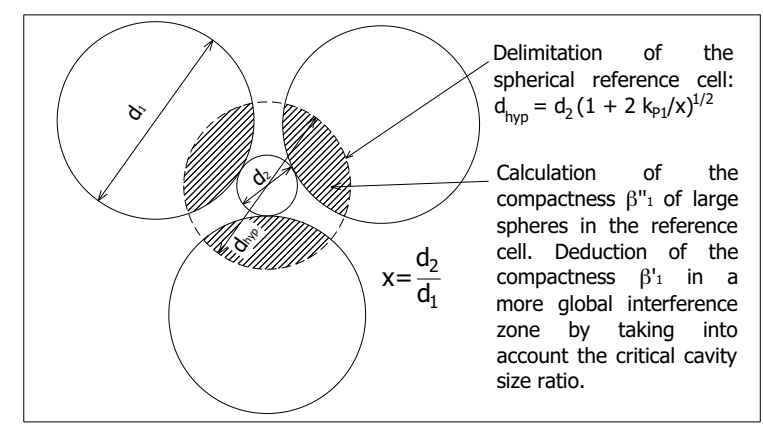

Fig. 4. Definition of the spherical reference cell for studying the loosening effect.

The diameter of the sphere delimiting the disturbed volume by the loosening effect is:

$$
d_{h y p}=d_{2} \sqrt{1+\frac{2 k_{P 1}}{x}}
$$

Let $\alpha_{1}$ be the real packing density of the large class 1 . We can deduce its virtual packing density $\beta_{1}$ :

$$
\beta_{1}=\alpha_{1} \frac{(1+K)}{K}
$$

The number of coarse spheres against a fine one is calculated from the Spherical Square Model (SSM):

$$
N_{21, S S M}^{\text {dense }}(x)=\frac{\pi(1+x)}{\arcsin \left(\frac{1}{1+x}\right)}
$$

The packing density $\beta_{1}$ of the coarse spheres in the reference cell is:

$$
\begin{aligned}
& \beta{ }_{1}(x)=\frac{\pi(1+x)}{4\left(1+\frac{2 k_{P 1}}{x}\right)^{\frac{3}{2}} \arcsin \left(\frac{1}{1+x}\right)} \times \\
& \times\left(2\left(1+\frac{2 k_{P 1}}{x}\right)^{\frac{3}{2}}-3\left(1+\frac{2 k_{P 1}}{x}\right)\left(1+\frac{k_{P 1}}{1+x}\right)+\left(1+\frac{k_{P 1}}{1+x}\right)^{3}-\frac{1}{x^{3}}+\frac{3 k_{P 1}}{x^{2}(1+x)}+\left(\frac{1}{x}-\frac{k_{P 1}}{1+x}\right)^{3}\right)
\end{aligned}
$$

The packing density of the large spheres in the more global interference zone is:

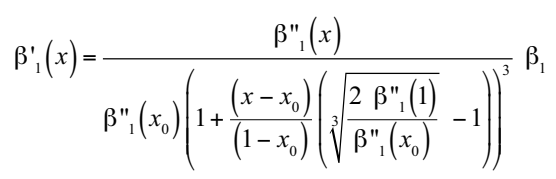

When $x=x_{0}, \beta^{\prime}{ }_{1}\left(x_{0}\right) / \beta_{1}=1$ : the loosening effect and the interference effect do not occur. When $x=1$, $\beta^{\prime}{ }_{1}(1) / \beta_{1}=0.5$ : the continuity between the "coarse dominant" field and the "fine dominant" field is provided when the volume fractions of each class are equal and when all particles become identical. The volume fraction of the fine particles at the "eutectic" point, corresponding to the crossing point into the "fine dominant" field, is:

$$
\phi_{2}^{*}(x)=\beta_{2}+\left(\left(1-\beta_{2}\right)\left(1-b_{21}(x)\right)-1\right) \beta_{1}^{\prime}(x)
$$

The loosening effect coefficient is deduced from this expression:

$$
a_{12}(x)=\frac{\beta_{1}-\beta_{1}^{\prime}(x)}{\phi_{2}^{*}(x)} \text { if } x \geq x_{0} \text { and } a_{12}(x)=0 \text { if } x \leq x_{0}
$$

$\mathrm{k}_{\mathrm{P} 1}$ remains to be determined by respecting the boundary condition $a_{12}(1)=1$ leading to solve the equation (9) by replacing $\mathrm{k}_{\mathrm{P} 2}$ by $\mathrm{k}_{\mathrm{P} 1}$ and $\beta_{2}$ by $\beta_{1}$.

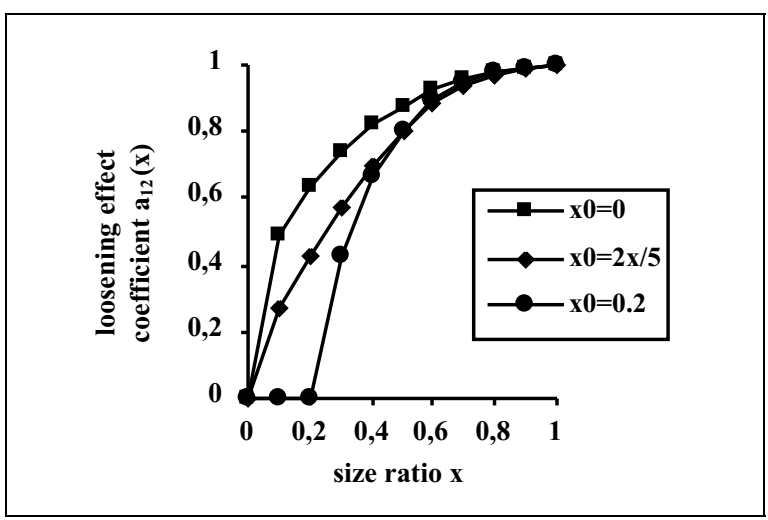

Fig. 5. ROAD theory: $\alpha_{1}=0.641-$ Vibration + compression.

\subsection{Compaction index $\mathrm{K}$ and critical cavity size ratio $\mathbf{x}_{0}$}

$K$ (Table 2) and $x_{0}$ (Table 3 ) need now to be calibrated by an analysis on 780 values on binary mixtures. 
$x_{0}$ depends on the shape and on the finish surface of the materials. For equivalent sizes, the loosening effect created by a small particle in a matrix of coarse ones is more important when this particle is irregularly shaped.

Table 2. Compaction index $K$

\begin{tabular}{|c|c|c|c|c|}
\hline $\begin{array}{l}\text { Packing } \\
\text { process }\end{array}$ & 占 & 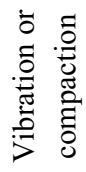 & 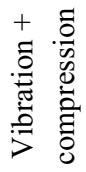 & 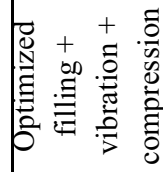 \\
\hline $\boldsymbol{K}$ & 4.7 & 5.6 & 9 & 15 \\
\hline
\end{tabular}

Table 3. Critical cavity size ratios $x_{0}$ ( $x$ : size ratio)

\begin{tabular}{|c|c|c|c|c|c|}
\hline \multirow[b]{2}{*}{$\begin{array}{c}\text { Type of } \\
\text { particles }\end{array}$} & \multicolumn{3}{|c|}{ Crushed particles } & \multirow[b]{2}{*}{ 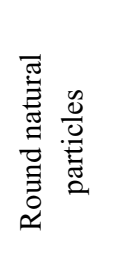 } & \multirow{2}{*}{ 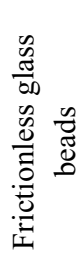 } \\
\hline & 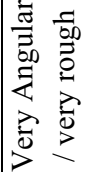 & 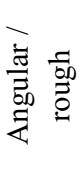 & $\begin{array}{l}\frac{\vec{\Xi}}{\vec{E}} \\
\stackrel{\sigma_{0}}{Z}\end{array}$ & & \\
\hline$x_{0}$ & $\mathbf{0}$ & 0.02 & 0.1 & $x_{0}=2 x / 5$ & 0.2 \\
\hline
\end{tabular}

The 4-parameter CPM has been evaluated [7] by comparison with 300 values of packing densities on frictionless glass beads, 20 on spherical particles numerically simulated, 125 on round natural particles and 335 on crushed particles (Table 4).

Table 4. Mean deviation $\xi$ and correlation coefficient $r$. Comparison between different models.

\begin{tabular}{|c|c|c|c|c|c|c|}
\hline \multirow{2}{*}{$\begin{array}{c}\text { Models } \\
\text { Materials } \\
\begin{array}{c}\mathbf{7 7 8 0} \\
\text { values) }\end{array}\end{array}$} & \multicolumn{2}{|c|}{$\begin{array}{c}\text { Original CPM } \\
\text { [1] }\end{array}$} & \multicolumn{2}{|c|}{ 3PPM [2, 3] } & \multicolumn{2}{|c|}{$\begin{array}{c}\text { 4-parameter } \\
\text { CPM [4, 7] }\end{array}$} \\
\cline { 2 - 7 } & $\boldsymbol{\xi}$ & $\boldsymbol{\xi}$ & $\mathbf{r}$ & $\boldsymbol{\xi}$ & $\mathbf{r}$ \\
\hline Glass beads & 0.012 & 0.9754 & 0.009 & 0.9863 & $\mathbf{0 . 0 0 7}$ & $\mathbf{0 . 9 9 0 4}$ \\
\hline Simulation & 0.012 & 0.8783 & 0.008 & 0.9598 & $\mathbf{0 . 0 0 6}$ & $\mathbf{0 . 9 8 7 7}$ \\
\hline $\begin{array}{c}\text { Round } \\
\text { natural }\end{array}$ & 0.009 & 0.9619 & 0.012 & 0.9534 & $\mathbf{0 . 0 0 7}$ & $\mathbf{0 . 9 7 8 8}$ \\
\hline Crushed & 0.013 & 0.9408 & 0.013 & 0.9455 & $\mathbf{0 . 0 1 0}$ & $\mathbf{0 . 9 6 4 2}$ \\
\hline
\end{tabular}

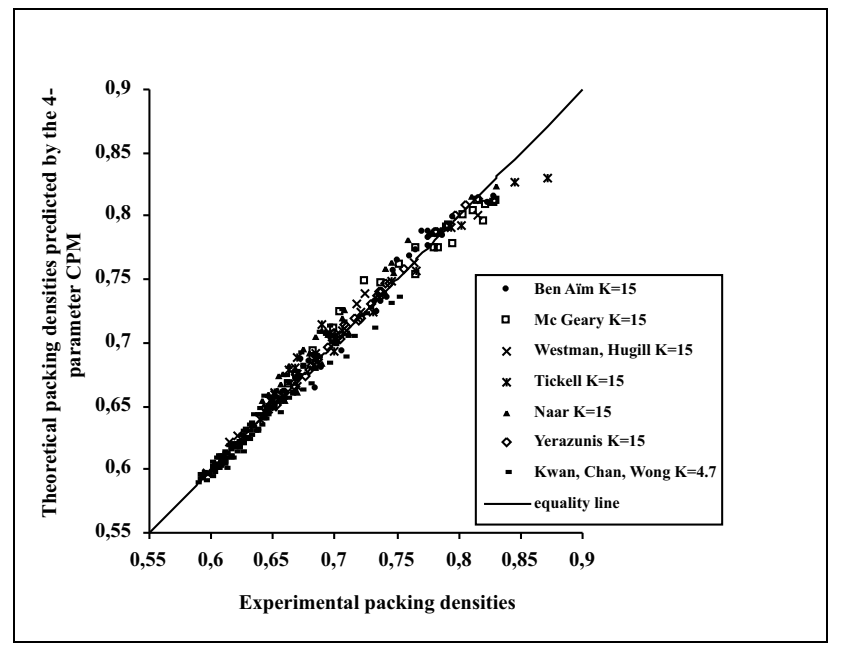

Fig. 6. Theoretical predictions by the 4-parameter CPM for frictionless glass beads with $x_{0}=0.2$.
The 4-parameter $C P M$ has been compared with the original CPM [1] and with the 3-parameter particle packing model (3PPM) [2, 3] (Table 4). It provides reliable predictions, as well as for low and high packing densities, especially at the optimum.

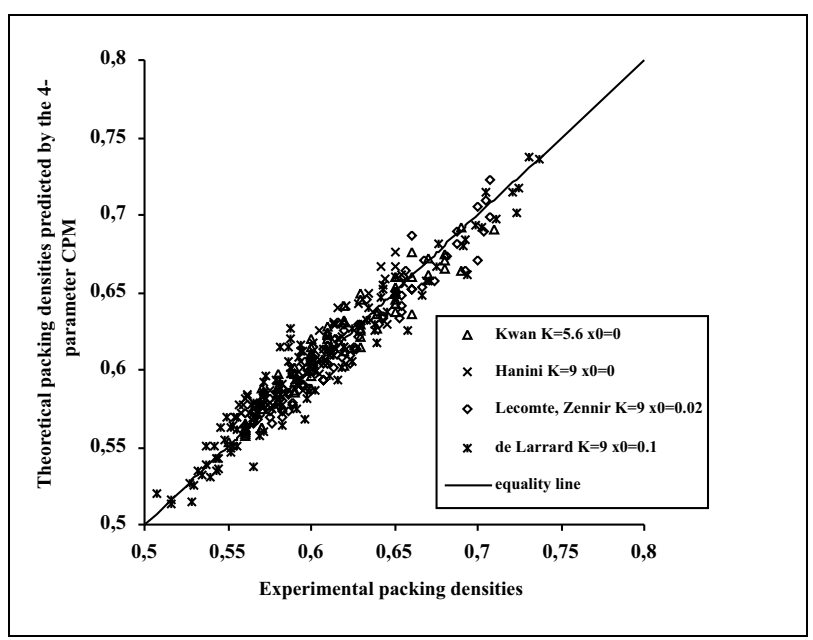

Fig. 7. Theoretical predictions by the 4-parameter CPM for crushed particles.

\section{Conclusion}

The 4-parameter CPM offers a new theory on the wall effect and on the loosening effect studied by considering elementary juxtaposed cells. The effect of a coarse or a fine particle is then examined on the basis of a foreign sphere surrounded by dominant class neighbours. It includes the introduction of a critical cavity size ratio depending on the type of materials. This reveals that there would not be a single curve for the geometrical interactions. The limitations of the proposed model are of two types. Firstly, the interaction coefficients are determined by considering the same virtual packing density for each granular class. Secondly, it remains to characterize the shape, the angularity and the surface texture of the particles by reference to test methods. However, the results obtained suggest that efforts will not have been in vain.

\section{References}

1. F. de Larrard, Concrete mixture proportioning: a scientific approach, E \& FN Spon, London (1999)

2. A.K.H. Kwan, K.W. Chan, V. Wong, Powder Technol. 237 (2013) 172-179

3. A.K.H. Kwan, V. Wong, W.W.S. Fung, Powder Technol. 274 (2015) 154-162

4. G. Roquier, Powder Technol. 302 (2016) 247-253

5. O. Koutný, J. Kratochvíl, J. Švec, J. Bednárek , Procedia Eng. 151 (2016) 198-205

6. Y. Knop, A. Peled, Constr. Build. Mater. 102 (2016) 44-50

7. G. Roquier, Etude de la compacité optimale des mélanges granulaires binaires: classe granulaire dominante, effet de paroi, effet de desserrement, Thèse soutenue à l'Université Paris-Est le 15 Fevrier 2016 